\title{
Molecular Docking Studies of Type III Secretion System Effector SopB Homolog in Vibrio vulnificus
}

Rama Adiga, Iddya Karunasagar, Indrani Karunasagar*

Department of Fishery Microbiology, Karnataka Veterinary, Animal and Fisheries Sciences University, College of Fisheries, Mangalore - 575 002, India

\begin{abstract}
Pathogenic bacteria use the needle shaped Type III secretion system to inject effector proteins into the host cell. The SopB effector protein of Salmonella mediates invasion by evading the host immune response. Being a phosphoinositide phosphatase, it synthesizes phospholipids at the host cell membrane, after targeting host cell ubiquitin. Ubiquitination of SopB are known to control the biological activity of SopB at the plasma membrane. The identified SopB effector protein of Vibrio vulnificus which is a human pathogen found in the marine environment was homologous to SopB of Salmonella and E.coli. Structural superposition with available structure of SopB of Salmonella yielded a DNA linking domain similar to Salmonella SopB. Ubiquitination sites for SopB homolog in V.vulnificus was predicted by bioinformatics tools which was further supported by molecular docking studies. The ubiquitin binding sites were proposed to be structurally similar to the conserved ubiquitin binding motif of human polymerase complexed with ubiquitin (2KTF). The ubiquitin binding sites having Leu residue at 226, Leu 235 and Leu 234 were conserved whereas the hydrophobic Phe was replaced by Tyr at 223 in the sopB homolog of V.vulnificus. The conserved Glu 228 of SopB protein was predicted to be involved in imparting a electronegative potential in the ligand binding site. The ubiquitin molecule docked with SopB of V.vulnificus had Leu8 for binding interaction and recognition which was found to be similar to the ubiquitin-human polymerase complex. Thus the host immune response was predicted to be targeted by SopB effector in V.vulnificus by altering the ubiquitin pathway.
\end{abstract}

Keywords: Vibrio vulnificus; SopB; Type III secretion system; Virulence; Ubiquitin; Protein-protein docking; Infection

\section{Introduction}

V. vulnificus is a gram-negative bacteria that is highly invasive. It has been isolated from marine environments from all round the world. Individuals who come in direct contact with the water infected with the organism through soft tissue exposure or those who consume contaminated sea food such as fish, oysters etc are at risk. The symptoms include high fever, gastroenteritis with high mortality rates caused by septicemia as described by Todar (http://textbookofbacteriology.net/V. vulnificus.html). Studies to better understand the pathogenesis and virulence of the microorganism are going on at a rapid pace [1]. The Type III Secretion System (TTSS) is a needle shaped apparatus used by pathogens to colonize the susceptible hosts. The needle pierces the host to release the toxic effectors into the host. The injected virulence effector proteins collectively inhibit the immune response of the host and aid in establishing infection [2]. Each effector protein has different targets in the host leading to progression of the disease. In some gram-negative organisms, the effector proteins disable the host cytoskeleton leading to cell necrosis. The host targets of only a few pathogens are known. Some pathogens target the host signaling pathway like the ubiquitin pathway. Ubiquitin is a crucial regulator of many host cellular processes and can modulate immune response [2]. The Salmonella outer protein, SopB effector of Salmonella spp. and the ExoU of Pseudomonas spp. target ubiquitin. Thus, targeting the ubiquitin machinery is important for virulence of the pathogen (Knodler et al., 2009). No study has been done on TTSS of V.vulnificus. In the present study we identify the SopB homolog of V.vulnificus and predict its 3D structure. We compare the obtained model of SopB of V.vulnificus with the structure of SopB of Salmonella. We also explore the possibility of ubiquitination of the SopB homolog of V.vulnificus using bioinformatics tools. Hence a ubiquitin molecule would be docked to the model of V.vulnificus. The proposed ubiquitin binding domain of V.vulnificus would be compared with the available structure of the ubiquitin binding domain. The predicted ubiquitin interacting domain of the SopB homolog may contribute to virulence of the organism.

\section{Materials and Methods}

NCBI blast was used to identify the homolog in V.vulnificus. The sequence of the protein SopB from V.vulnificus was modeled by ITasser server [4] (Zhang labzhang.bioinformatics.ku.edu/I-TASSER/). The structure was energy minimized using Deepview and the refined structure was validated structurally for anolea using the web address http://swissmodel.expasy.org/ [5] and Verify 3D server http://nihserver. mbi.ucla.edu/Verify_3D/ [6]. The Vadar version 1.8 (http://redpoll. pharmacy.ualberta.ca/vadar/ ) was used for analyzing and assessing protein structures [7]. Prediction of ubiquitination was done using the server (http://iclab.life.nctu.edu.tw/ubipred) [8]. Pairwise Dalite was used for superimposition of structures (http://ekhidna.biocenter. helsinki.fi/dali_server/). Root Mean Square Inner Product (R.M.S.I.P) for protein binding with similar low energy modes was calculated by using the Aladyn server (http://aladyn.escience-lab.org/index.php). The validated homology modeled structure was used for proteinprotein docking. Docking was done using the PATCHDOCK server [9]. Surface potential was generated using Adaptive Poisson-Boltzmann Solver (A.B.P.S) electrostatics. The selected model was viewed with pymol 0.9 (Delano scientific) or chimera.

*Corresponding author: Dr. Indrani Karunasagar, Professor \& Head, Department of Fishery Microbiology, Karnataka Veterinary, Animal and Fisheries Sciences University College of Fisheries, Mangalore, India, Tel: +91 824 2246384; Fax: +91 824 2246384; E-mail: karuna8sagar@yahoo.com; mircen@sancharnet.in

Received November 22, 2010; Accepted January 28, 2011; Published February 10, 2011

Citation: Adiga R, Karunasagar I, Karunasagar I (2011) Molecular Docking Studies of Type III Secretion System Effector SopB Homolog in Vibrio vulnificus. J Comput Sci Syst Biol 4: 016-020. doi:10.4172/jcsb.1000070

Copyright: (c) 2011 Adiga R, et al. This is an open-access article distributed under the terms of the Creative Commons Attribution License,which permits unrestricted use, distribution, and reproduction in any medium, provided the original author and source are credited. 
Citation: Adiga R, Karunasagar I, Karunasagar I (2011) Molecular Docking Studies of Type III Secretion System Effector SopB Homolog in Vibrio vulnificus. J Comput Sci Syst Biol 4: 016-020. doi:10.4172/jcsb.1000070

\section{Results and Discussion}

\section{Sequence analysis}

The SopB homologue in V.vulnificus was identified by NCBI blast and had the accession number NP_763080. It was 53\% similar to SopB from Salmonella enterica and 52\% similar to SopB from Escherichia coli (Figure1). SopB belonged to the ParB superfamily of type III secretion system effector protein which generates many active inositol phospholipids and inositol phosphates, to modulate host cytoskeleton. The SopB family protein in Salmonella has flexibly linked regions

\section{E. coli \\ salmone 1

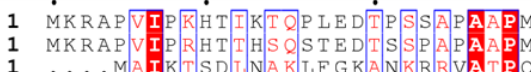

Figure 1: Multiple seq alignment of SopB from E.coli, Salmonella and V.vulnificus.

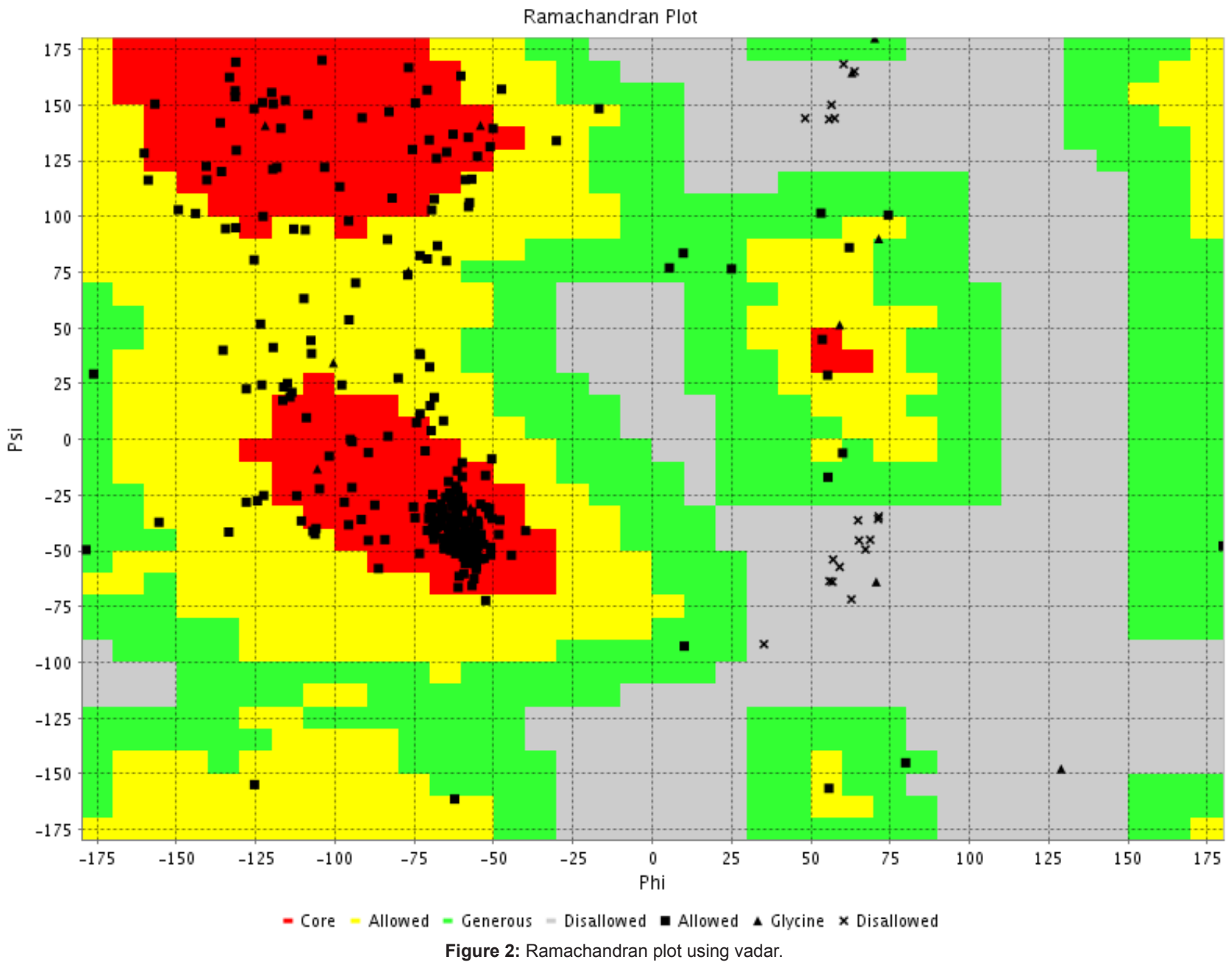


which bind DNA to achieve partition of chromosome. Like all effector proteins a signal-peptide is located at the amino terminal end of the protein which is not conserved. Guttman and coworkers observed the presence of aliphatic (Ile, Leu, Val) or proline residues at amino acid third or fourth position in effectors of Pseudomonas [10]. The above criteria were found in the SopB homolog of V.vulnificus since isoleucine was present at position three. Amphipathic residues have also been observed in the SopB homolog of V.vulnificus consisting of many lysine residues in the first twenty amino acid residues. This may contribute to the amphipathic nature of the signal as observed by Lloyd and coworkers [11]. The identified SopB homolog of V.vulnificus met with the criteria described by various workers [10-12]. Sequence analysis yielded iron binding motif exemplified by the ExxxE motif as reported by Wosten and coworkers [13] and was predicted at position number 69-73. The iron binding motifs were also observed in SopB protein of Salmonella and E.coli. Prediction of ubiquitination by Ubipred server revealed the presence of probable sites of ubiquitination of the protein. Calcium binding motif exemplified by the DxxD motif was found at

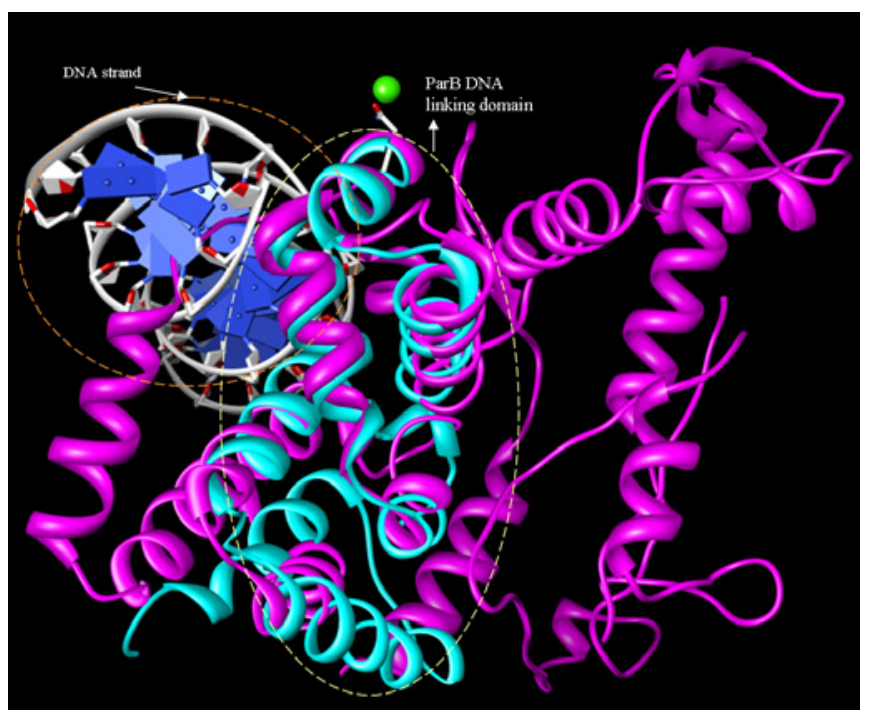

Figure 3: The superposed structure of Salmonella SopB 3MKZ:A(cyan) with model SopB(magenta).ParB domain of SopB fragment in Salmonella showing DNA bridging effect.

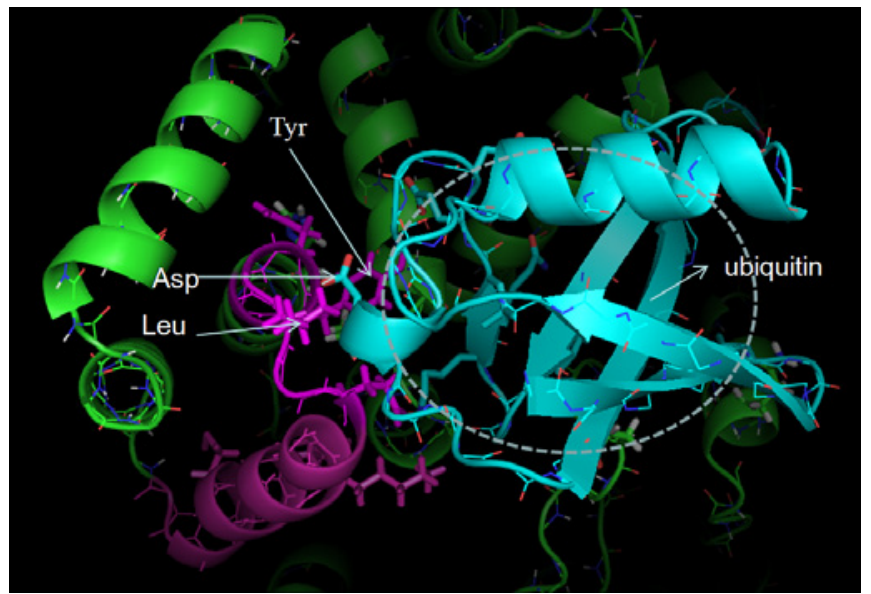

Figure 4: ubiquitin binding domain (magenta)showing hydrophobic Tyr 223 and Leu 226.

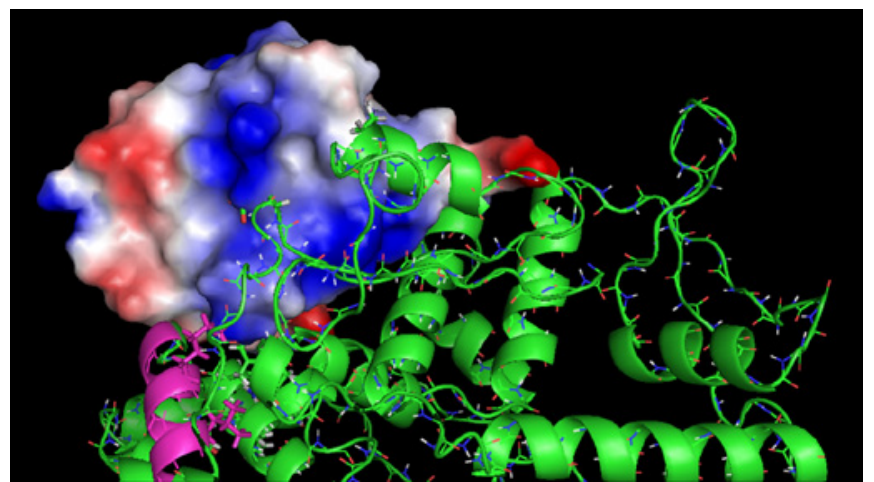

Figure 5: Overall positive electrostatic potential (shown in blue) of ubiquitin surface.

position 170-173. The DxxD motif thought to be the calcium binding site [14] was predicted to be important since calcium is a cofactor for the activity of the inositol phosphatases. Calcium binding motifs were observed in SopB of both the sequences of Salmonella and E.coli spp.. The carboxy terminal of the protein at position 300-304 was predicted to have the repression domain with the LxLxL motif (Figure 1). The motif is not conserved in the other SopB homologs. Pauw and coworkers described the repressor domain in most zinc finger motifs of DNA binding protein as having the LxLxL motif [15].

\section{Structural analysis of SopB}

The 3D structure of the protein SopB from V.vulnificus was modeled using I Tasser (http://zhanglab.ccmb.med.umich.edu/I-TASSER). After refinement and validation of the structure the ramachandran plot analysis revealed the protein to have $80 \%$ residues in favoured region, $14.3 \%$ in allowed region and $5.3 \%$ in outlier region (Figure 2).The structure of SopB from V.vulnificus was compared with the available structure of SopB from Salmonella. Structural superimposition of SopB from Salmonella and V.vulnificus gave an RMSD of $4.5 \mathrm{~A}^{\circ}$. The chainA fragment of Salmonella SopB from residues 155 to 272, harbour a DNA linking site (Figure 3). The flexible attachment of DNA helps the SopB protein give a partition effect to DNA and enable binding of several other proteins. The superimposition study shows a similar DNA dimerization site which may be present in SopB homolog of $V$. vulnificus.

\section{Docking of SopB effector with ubiquitin}

Knodler and co-workers have shown the SopB of Salmonella attacks the ubiquitin machinery by conjugation with ubiquitin. The strategy is employed by pathogenic bacteria to manipulate and overpower the host. Bioinformatic tools helped predict the ubiquitination of the SopB protein in V.vulnificus. We use docking studies to support the hypothesis that a similar mechanism of host manipulation exists in $V$ vulnificus. Molecular docking of the SopB model with ubiquitin as a ligand (PDB: 1UBQ) was used in a protein-protein docking study. Based on desolvation energy, interfacial energy, the docking results with Patchdock were obtained. The atomic contact energy obtained for the binding was +317 . The R.M.S.I.P for binding ubiquitin and ubiquitin like proteins vary from 0.5 to 0.7 [16]. The R.M.S.I.P for the above protein found was 0.6 which is in the range. Tyrosine $\mathrm{Y}^{223}$ and leucine $\mathrm{L}^{226}$ (conserved) were the probable hydrophobic residues interacting with ubiquitin (Figure 4). Ubiquitin had a largely electronegative surface docking on to the SopB protein (Figure 5). Figure 6 shows the docked ubiquitin stabilized by acidic patches to ubiquitin. Ubiquitin binding was compared with the 3D structure of complexes downloaded 
Citation: Adiga R, Karunasagar I, Karunasagar I (2011) Molecular Docking Studies of Type III Secretion System Effector SopB Homolog in Vibrio vulnificus. J Comput Sci Syst Biol 4: 016-020. doi:10.4172/jcsb.1000070

from PDB. It was found to be comparable to the ubiquitin interacting structure of human polymerase obtained from the PDB (code: $2 \mathrm{KTF}$ chainB). Chain $\mathrm{B}$ containing the conserved ubiquitin binding motif structurally superimposed with the SopB homolog with an RMSD of $4.2 \mathrm{~A}^{\circ}$. Figure 7 shows the superimposed image of ubiquitin binding domain of human polymerase (with ubiquitin removed) and the sopB protein. The conserved ubiquitin interaction domain consists of two helices separated by a trans- proline [17].The comparison leads to the sequence alignment of the binding domain (Figure 7). The alignment showed ubiquitin binding sites having Leu residue at 226, Leu 235 and Leu 234 which were conserved. The hydrophobic Phe was replaced by Tyr at 223. The hydrophobic Cys residue at 238 was present instead of the hydrophobic tryptophan near the $\mathrm{C}$ term of the domain. Figure 8 gives

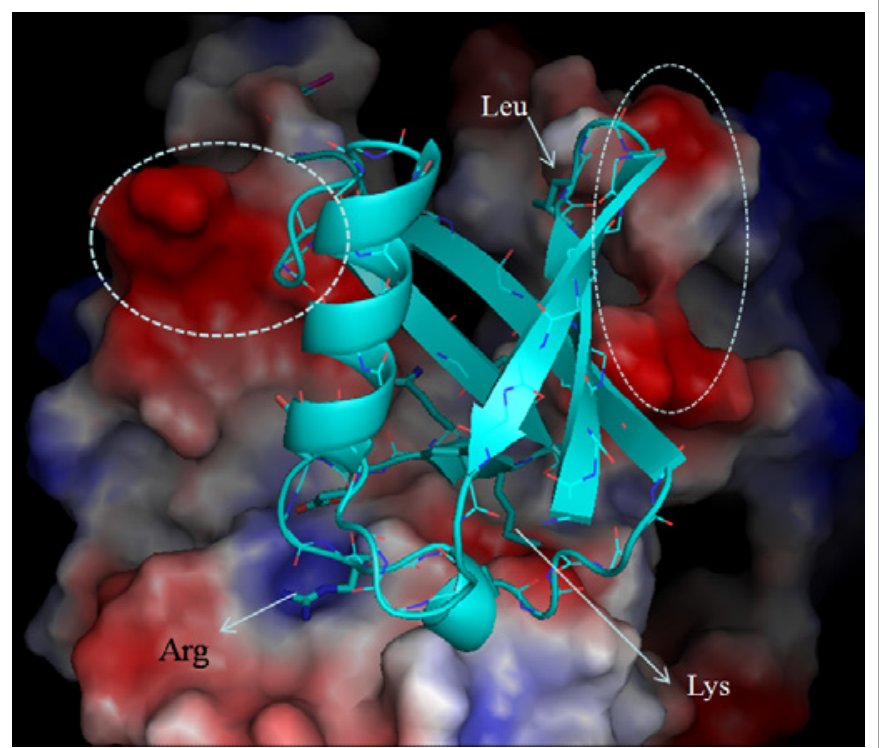

Figure 6: Red patches with electronegative potential may stabilize the ubiquitin (cyan colored) molecule. Arg ${ }^{54}$ dock on the positive potential surface while Lys ${ }^{48}$ and Leu ${ }^{8}$ dock on the negative potential of the protein molecule attracting the sopB homolog.

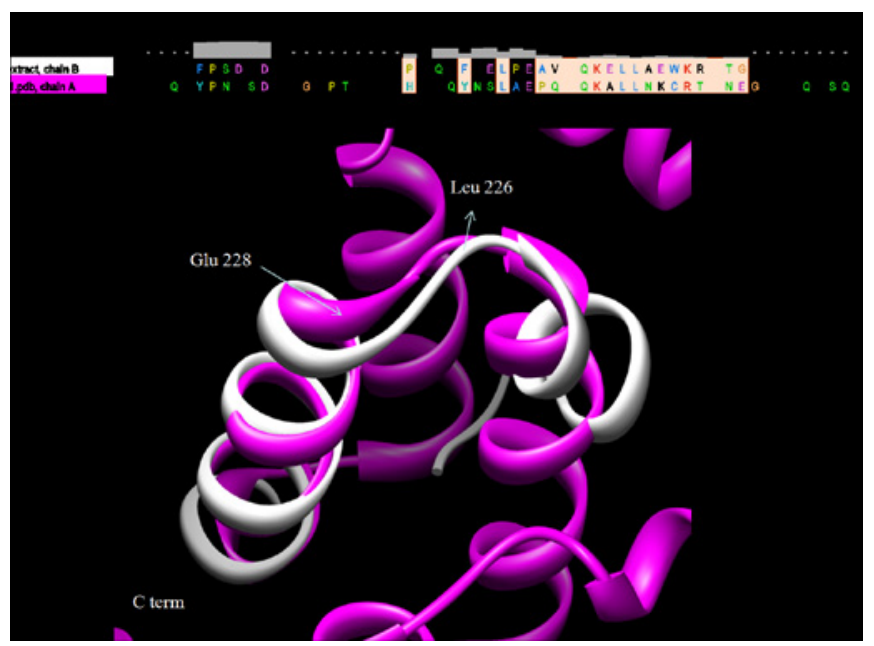

Figure 7: Superimposition of structure of ubiqutin complexed human polymerase (PDB: 2KTF chainB colored white) with modelled sopB homolog of V.vulnificus (colored magenta).Above: Sequence alignment of the proposed ubiquitin interacting motif of SopB in V.vulnificus and conserved ubiquitin-binding motif (UBM2) of Y-family polymerase of human.

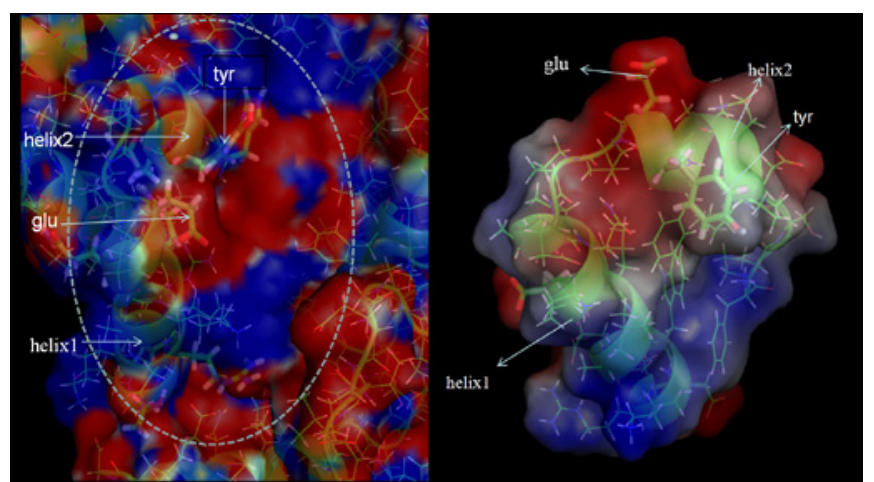

Figure 8: Comparitive surface potential of V.vulnificus proposed ubiquitin binding domain (left) and conserved ubiquitin-binding motif (UBM2) of Y-family polymerase of human (right)

a comparison of surface potential generated by A.B.P.S electrostatics. The conserved glutamic acid (E 228) may provide the negative surface potential in the ligand binding site as seen in the ubiquitin interacting domain of the human polymerase (Figure 8). Thus the effector protein of V.vulnificus was predicted to target ubiquitin of host. The predicted ubiquitin binding site from 220-242 with conserved Leu ${ }_{226}$ and Glu was found suitable to be tested in the wet lab for any pharmacological intervention that may be carried out.

\section{Conclusion}

The type III secretion effector SopB in V.vulnificus was homologous to Salmonella SopB and had conserved DNA linking domain typical of ParB family. The target of the protein may be the ubiquitin machinery as proposed by docking studies. The ubiquitin interacting domain was found similar to the human polymerase ubiquitin complex which was structurally conserved. This finding may throw light on the similar mechanism of targeting the host as Salmonella.

\section{Acknowledgments}

The funding support by the Dept. of Biotechnology, Bioinformatics centre Govt. of India is gratefully acknowledged.

\section{Disclosure}

The authors report no conflicts of interest in this work.

\section{References}

1. Jones MK, Oliver JD (2009) Vibrio vulnificus: disease and pathogenesis. Infect Immun 77: 1723-1733.

2. Ghosh P (2004) Process of protein transport by the type III secretion system Microbiol Mol Biol Rev 68: 771-795.

3. Majetschak M (2010) Extracellular ubiquitin: immune modulator and endogenous opponent of damage-associated molecular pattern molecules. J Leukoc Biol.

4. Zhang $Y$ (2008) I-TASSER server for protein 3D structure prediction. BMC Bioinformatics 9: 40. Melo F, Feytmans E (1997) Novel knowledge-based mean force potential at atomic level. J Mol Biol 267: 207-222.

5. Lüthy R, Bowie JU,Eisenberg D (1992) Assessment of protein models with three-dimensional profiles. Nature 356: 83-85.

6. Willard L, Ranjan A, Zhang H , Monzavi H, Boyko R F, et al. (2003) VADAR: a web server for quantitative evaluation of protein structure quality. Nucleic Acids Res. $31: 3316-3319$

7. Tung CW, Ho SY (2008) Computational identification of ubiquitylation sites from protein sequences BMC Bioinformatics. 9: 310.

8. Duhovny SD, Inbar Y, Nussinov R, Wolfson HJ (2005) PatchDock and SymmDock: servers for rigid and symmetric docking. Nucl Acids Res 33 : W363-367. 
Citation: Adiga R, Karunasagar I, Karunasagar I (2011) Molecular Docking Studies of Type III Secretion System Effector SopB Homolog in Vibrio vulnificus. J Comput Sci Syst Biol 4: 016-020. doi:10.4172/jcsb.1000070

9. Guttman DS, Vinatzer BA, Sarkar SF, Ranall MV, Kettler G, et al. (2002) A Functional Screen for the Type III (Hrp) Secretome of the Plant Pathogen Pseudomonas syringae. Science 295:1722-1726.

10. Lloyd SA, Norman M, Rosqvist R, Watz WH (2001) Yersinia YopE is targeted for type III secretion by N-terminal, not mRNA, signals. Mol Microbiol 39: 520 531

11. Schechter LM, Roberts KA, Jamir Y, Alfano JR, Collmer A (2004). Pseudomonas syringae type III secretion system targeting signals and novel effectors studied with a Cya translocation reporter. J Bacteriol 186: 543-55.

12. Wosten MM, Kox LF, Chamnongpol FS, Soncini FC, et al. (2000) A signa transduction system that responds to extracellular iron. Cell 103: 113-125.

13. Handschuh G, Luber B, Hutzler P, Höfler H, Becker K-F (2001) Single amino acid substitutions in conserved extracellular domains of E-cadherin differ in their functional consequences. J Mol Biol 314: 445-454.

14. Pauw B, Hilliou FAO, Martin VS, Chatel G , Wolf CJF, et al. (2004) Zinc finger proteins act as transcriptional repressors of alkaloid biosynthesis genes in Catharanthus roseus. J Biol Chem 279: 52940-52948.
15. Potestio R, Aleksiev T, Pontiggia F, Cozzini S, Micheletti C (2010) ALADYN: a web server for aligning proteins by matching their large-scale motion. Nucleic Acids Res 38: W41-45.

16. Cui G, Benirschke RC, Tuan HF, Juranic N,Macura S, et al. (2010) Structural basis of ubiquitin recognition by translesion synthesis DNA polymerase. Biochemistry $49: 10198-207$.

17. Comeau SR, Gatchell DW, Vajda S, Camacho CJ (2004) ClusPro: an automated docking and discrimination method for the prediction of protein complexes. Bioinformatics 20: 45-50.

18. Hicke L, Dunn R (2003) Regulation of membrane protein transport by ubiquitin and ubiquitin-binding proteins. Annu Rev Cell Dev Biol 19: 141-72.

19. Kozakov D, Hall DR, Beglov D, Brenke R, Comeau SR, et al. (2010) Achieving reliability and high accuracy in automated protein docking: Cluspro, PIPER, SDU, and stability analysis in CAPRI rounds 13-19. Proteins 78: 3124-3130.

20. Todar K (2008) Todar's Online Textbook of Bacteriology. 